\title{
The influence of longitudinal density variation in coronal loops on the eigenfunctions of kink-oscillation overtones
}

\author{
J. Andries ${ }^{1,2, \star}$, I. Arregui ${ }^{3}$, and M. Goossens ${ }^{1}$ \\ 1 Centrum voor Plasma Astrofysica and Leuven Mathematical Modelling and Computational Science Centre, KULeuven, \\ Celestijnenlaan 200B, 3001 Leuven, Belgium \\ e-mail: [Jesse. Andries; Marcel. Goossens] @wis . kuleuven.be \\ 2 Centre for Stellar and Planetary Astrophysics, Monash University, 3800 Victoria, Australia \\ 3 Departament de Física, Universitat de les Illes Balears, 07122 Palma de Mallorca, Spain \\ e-mail: inigo .arregui@uib.es
}

Received 8 December 2008 / Accepted 24 January 2009

\section{ABSTRACT}

\begin{abstract}
Context. As coronal loops are spatially at least partially resolved in the longitudinal direction, attempts have been made to use the longitudinal profiles of the oscillation amplitudes as a seismological tool.

Aims. We aim to derive simple formulae to assess which oscillation modes and which quantities of the oscillation (displacement or compression) are most prone to modifications induced by stratification of the equilibrium density along the loop. We furthermore clarify and quantify that the potential of such a method could be enhanced if observational profiles of the compression in the oscillations could be determined.

Methods. By means of a linear expansion in the longitudinal stratification along with the "thin tube" approximation, the modifications to the eigenfunctions are calculated analytically. The results are validated by direct numerical computations.

Results. Higher axial overtones are found to be more affected by equilibrium stratification and hence would provide a much better tool if observed. For the $k-1$ th overtone the compression is found to be around $(k+2)^{2} / k^{2}$ times more sensitive to longitudinal density variation than the displacement. While the linear formulae do give a good indication of the strength of the effects of longitudinal density stratification, the numerical computations indicate that the corrections to the approximate analytical results are significant and cannot be neglected under the expected coronal conditions.
\end{abstract}

Key words. magnethohydrodynamics (MHD) - waves - Sun: corona - Sun: oscillations - Sun: magnetic fields

\section{Introduction}

The clear detection of flare generated coronal loop oscillations by the Transition Region and Coronal Explorer has initiated the field of coronal loop seismology. An interpretation of the oscillations in terms of the fundamental kink oscillation of a coronal flux tube (as treated by Edwin \& Roberts 1983) enabled investigators to deduce a rough estimate for the coronal magnetic field strength from the observed periodicity (Nakariakov 2000; Nakariakov \& Ofman 2001). Ruderman \& Roberts (2002) subsequently extended the basic model to include smooth transversal density variations which give rise to a continuous spectrum and associated continuum damping, also known as Alfvén resonant absorption. Within that framework the additional model parameter that quantifies the transversal density inhomogeneity length scale can directly be related to the damping time of the oscillations (Goossens et al. 2002). In recent studies by Arregui et al. (2007) and Goossens et al. (2008) the third remaining parameter of the density contrast between the loop and the surrounding plasma (which was assigned an ad hoc value in all previous studies) was taken into account consistently.

The simultaneous detection of axial overtones of a coronal loop kink-mode oscillation by Verwichte et al. (2004) has triggered an increased interest for theoreticians to refine the theoretical modelling of transverse coronal loop oscillations, and has greatly advanced coronal seismology (for reviews see

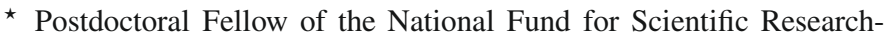
Flanders (Belgium) (FWO-Vlaanderen).
Nakariakov \& Verwichte 2005; Roberts 2008). With regard to the potential of coronal seismology, the discovery of harmonic coronal loop oscillations has raised awareness on two important issues.

Firstly, the simultaneous detection of several oscillation frequencies is an important advance in the development of any seismological endeavour. It allows us to add complexity to the proposed theoretical models and infer values for the additional parameters without detailed spatial resolution of the oscillations. In a coronal context, this was first illustrated by Andries et al. (2005a) by means of the computations of eigenfrequencies and eigenmodes in coronal loop models with longitudinal density stratification (Andries et al. 2005b) (using a method similar to that of Díaz et al. 2002, 2004). The deviation of the ratio of the fundamental period to the successive overtones from $1 / 2,1 / 3$, etc. was related to, and hence used as a measure for, the density scale height. McEwan et al. (2006) investigated alternative explanations for the anomalous period ratio but concluded that it is most probably due to longitudinal density stratification (or magnetic field variation, which had not been assessed at that time). Meanwhile much more theoretical as well as observational work has been done along those lines (Donnelly et al. 2006; Dymova \& Ruderman 2006b,a; Díaz et al. 2006; Van Doorsselaere et al. 2007; De Moortel \& Brady 2007; Díaz et al. 2007; McEwan et al. 2008). Verth \& Erdélyi (2008) and Ruderman et al. (2008) included the variation of the magnetic field along the loop into the calculations. They concluded that it has an effect opposite to that of the density variation and may alter the 
seismologically inferred density scale heights considerably. By using observational estimates of the magnetic field expansion Verth et al. (2008) performed a refined seismological analysis of the density scale height by means of the period ratio.

Secondly, since the identification of the first overtone in the observations was not only made because of its frequency close to twice that of the fundamental, but also by observational evidence that the amplitude of that mode had maxima halfway down the loop legs and a node at the loop top, this made it clear that at least in the axial direction we are able at present to partly resolve the spatial structure of the oscillations. It was strongly emphasised by Erdélyi \& Verth (2007) and Verth et al. (2007) that the longitudinal spatial resolution could possibly allow for the estimation of loop model parameters based on the deviations in the eigenfunctions rather than the eigenfrequencies. The effect of longitudinal density stratification on the transversal displacement amplitude was however found to be not particularly strong for the fundamental oscillation (Erdélyi \& Verth 2007; Safari et al. 2007), and due to several uncertainties in the observations (projection effects, localisation of the loop footpoints), probably not detectable at present with reasonable confidence. For the first overtone however it was found that the shift of the antinodes towards the footpoints is much more pronounced and could possibly be detectable. Progress in these detections could possibly be sought in advanced edge tracking detection methods as used by Jess et al. (2008).

The present paper aims at contributing to this second point.

We derive simple algebraic expressions that quantify how much the eigenfunctions are altered by the presence of a longitudinal density variation. In order to arrive at analytical expressions, we assume a relatively weak density structuring which is identical inside and outside the loop. The expressions are derived generally, valid for any harmonic, and both for the displacement and the compression. The derivation starts from a tube of arbitrary thickness but eventually specializes to a "thin tube" to obtain concise formulae. A similar formula (for the displacement only) was derived by Safari et al. (2007) starting directly from the "thin tube" formula by Dymova \& Ruderman (2005). They used it to validate the results about the modifications to the displacement profiles of the fundamental and its first harmonic by Erdélyi \& Verth (2007) and Verth et al. (2007). However, the formulae enable us to assess whether higher harmonics, if they could possibly be detected in the future, would have even higher potential for spatial seismology. The answer is clearly affirmative. However, it is debatable whether the shift of the antinodes is in general a good observational signature of the modifications to the eigenfunctions.

Secondly, the derivations also address a conclusion which was already made by Andries et al. (2005b) but which has not received much attention so far. From the numerical computations it was concluded that the influence on the eigenfunctions seems to be much more pronounced in the total pressure perturbation than in the transversal displacement although a quantitative analysis was not performed. Observationally however, it is the displacement which is measured. Being nearly incompressible, it is clear that kink modes are most easily detected as transverse displacements and measuring the compression in the observations is probably not easy to achieve. We want to argue however that from a theoretical perspective the potential for revealing information on the longitudinal structure by means of analysis of the spatial eigenfunctions is much greater if the compression or the density variation could be measured.

In Sect. 2 the approximate formulae for the modifications of the eigenmodes are derived analytically. Based on these formulae it is discussed in Sect. 3 how the different harmonics are influenced. In Sect. 4 the validity of these formulae is checked numerically.

\section{Model and analysis}

\subsection{Model and setup}

The loop model is exactly as in Andries et al. (2005b). The coronal loop is viewed as a straight cylindrical flux tube where the magnetic field is constant but the density varies both in the radial (hence characterising the tube as a density enhancement) and longitudinal direction. The slow waves are removed from the analysis as $\beta=0$ is an appropriate approximation in the lower corona. As before the observed oscillations are modelled as linear oscillations because the observed velocities are small compared to the local Alfvén speed. Within the linear treatment this is equivalent to the observation that the displacement is small compared to the length of the loop. However, in a different context, the nonlinear coupling between different azimuthal components has been related to the ratio between the displacement and the tube radius (Ruderman 1992). At present it is not clear how important this effect of non-linear coupling of azimuthal wave numbers is for standing kink oscillations.

In a cylindrical reference frame where the equilibrium quantities are independent of $\varphi$ and of time, the perturbed quantities can be set proportional to $\exp [\imath(m \varphi)]$ and $\exp [-l \omega t]$ because no coupling between different Fourier modes can occur. Here $\omega$ is the circular frequency and $m$ (an integer) is the azimuthal wave number. As we are interested in oscillations that displace the axis of the loop and also the loop as a whole, we need to take $m=1$. This is the only value of the azimuthal wave number for which the loop is displaced. For a loop that is uniform in the longitudinal direction the longitudinal variation of the kink oscillation can be described by one axial wave number $k_{z}$ with the axial behaviour proportional to $\sin \left(k_{z} z\right)$. For a loop of length $L, k_{z}=n \frac{\pi}{L}$, $n$ is a positive integer. $n=1$ corresponds to the fundamental axial mode; $n \geq 2$ correspond to the axial overtones. A consequence of longitudinal stratification is that a given mode cannot be described by one axial wave number but now instead an infinite sine series is required. Unless the longitudinal stratification is very strong, one term in this infinite series is dominant so that we can still speak about the fundamental axial mode etc. When we speak about the $k$ th mode in what follows, this is meant with respect to the axial ordering as we just discussed. The radial part of the spatial solutions of the modes does not follow a harmonic variation. However, the modes can be distinguished by the number of nodes in the radial part of their spatial solution. The kink oscillations invoked to explain the observed transverse oscillations in coronal loops have no nodes in the radial part of their spatial solution. They are fundamental radial kink oscillations.

Solutions to the linearised expressions can be obtained analytically in the internal and external regions where the density does not vary with radial position $r$. We follow the notation as introduced by Andries et al. (2005b), where the Eulerian pressure perturbation and the radial component of the Lagrangian displacement, both evaluated at the discontinuous boundary, are expressed as a sine series expansion (with $L$ the loop length):

$$
\begin{aligned}
& p_{\mathrm{T}}(r=R, z)=\sum_{k=1}^{+\infty} \sum_{n=1}^{+\infty} A^{k} \Pi_{n}^{k} \sin \left(\frac{n \pi}{L} z\right) \\
& \xi_{r}(r=R, z)=\sum_{k=1}^{+\infty} \sum_{n=1}^{+\infty} A^{k} \Xi_{n}^{k} \sin \left(\frac{n \pi}{L} z\right) .
\end{aligned}
$$


$\sum_{k=1}^{+\infty} A^{k} \Pi_{n}^{k}$ and $\sum_{k=1}^{+\infty} A^{k} \Xi_{n}^{k}$ simply represent the Fourier sine transformations in $z$ at $r=R$, whereas $\sum_{n=1}^{+\infty} \Pi_{n}^{k} \sin \left(\frac{n \pi}{L} z\right)$ and $\sum_{n=1}^{+\infty} \Xi_{n}^{k} \sin \left(\frac{n \pi}{L} z\right)$ are eigenmodes of the Alfvén differential operator in the $z$ direction. $\Pi_{n}^{k}$ and $\Xi_{n}^{k}$ can thus be seen as transformation matrices between two different orthogonal bases. However, they are not orthonormal in the sense that the basis vectors $\sum_{n=1}^{+\infty} \Pi_{n}^{k} \sin \left(\frac{n \pi}{L} z\right)$ and $\sum_{n=1}^{+\infty} \Xi_{n}^{k} \sin \left(\frac{n \pi}{L} z\right)$ are not of unit length. Later on superscripts in and ex will be added to these expressions to indicate that the values are obtained from a solution of the equations in the internal and external region, but where possible they are dropped for notational convenience.

As at the boundary surface $r=R$ both $\xi_{r}$ and $p_{\mathrm{T}}$ have to be continuous (in the radial direction) the dispersion relation is obtained as the determinant of the resulting infinite set of matching conditions. Without stratification $\Pi_{j}^{k}$ and $\Xi_{j}^{k}$ are zero whenever $k \neq j$, yielding a block diagonal system with a $2 \times 2$ block for each Fourier mode.

The equilibrium density is expressed as a constant (in the $z$ direction) plus a sine series expansion:

$\rho(r, z)=\rho_{0}(r)\left[1+\sum_{n=1}^{+\infty} \alpha_{n}(r) \sin \left(\frac{n \pi}{L} z\right)\right]$.

In Andries et al. (2005b) we have analysed in detail how the dispersion relation can be expanded linearly in $\alpha$, where $\alpha$ is a small parameter representing the amount of longitudinal stratification. We were thus able to determine the frequency shift due to stratification in the limit of small stratification. However, although we had also computed the eigenfunction numerically nonlinearly in $\alpha$, at that time we were unaware of the potential of analytic expressions for the variation of the eigenfunctions. We will pursue this in what follows. We drop the subscript on $\alpha$ for convenience and hence consider the influence of the contribution of a single Fourier mode in the equilibrium density profile (3). The results can be readily generalised afterwards.

\subsection{Analysis}

Consider the ratios of the $j$ th term and the $k$ th term in the sine series expansion (denoted as $R P_{k}^{j}$ and $R X_{k}^{j}$ for pressure and displacement) of the $k$ th eigenmode. To first order in $\alpha$ we obtain (with subscripts 0 and 1 representing the order in the $\alpha$ expansion):

$$
\begin{aligned}
\left((R P)_{k}^{j}\right)_{1} & =\left(\frac{\sum_{l=1}^{+\infty} A^{l} \Pi_{j}^{l}}{\sum_{l=1}^{+\infty} A^{l} \Pi_{k}^{l}}\right)_{1}=\frac{\left(\sum_{l=1}^{+\infty} A^{l} \Pi_{j}^{l}\right)_{1}}{\left(\sum_{l=1}^{+\infty} A^{l} \Pi_{k}^{l}\right)_{0}} \\
& =\frac{1}{A_{0}^{k} \Pi_{k 0}^{k}} \sum_{l=1}^{+\infty}\left(A_{0}^{l} \Pi_{j 1}^{l}+A_{1}^{l} \Pi_{j 0}^{l}\right), \\
& =\frac{1}{A_{0}^{k} \Pi_{k 0}^{k}}\left(A_{0}^{k} \Pi_{j 1}^{k}+A_{1}^{j} \Pi_{j 0}^{j}\right), \\
& =\frac{\Pi_{j 1}^{k}}{\Pi_{k 0}^{k}}+\frac{A_{1}^{j} \Pi_{j 0}^{j}}{A_{0}^{k} \Pi_{k 0}^{k}}
\end{aligned}
$$

and likewise for the displacement:

$$
\left((R X)_{k}^{j}\right)_{1}=\frac{\Xi_{j 1}^{k}}{\Xi_{k 0}^{k}}+\frac{A_{1}^{j} \Xi_{j 0}^{j}}{A_{0}^{k} \Xi_{k 0}^{k}}
$$

The expressions for $\Pi_{j}^{k}$ and $\Xi_{j}^{k}$ contain the factor $\psi_{j}^{k}$ and hence since $\psi_{j 0}^{k}=0$ whenever $j \neq k$ :

$$
\begin{aligned}
& \Pi_{j 1}^{k}=\Pi_{k 0}^{k} \frac{\psi_{j 1}^{k}}{\psi_{k 0}^{k}}, \\
& \Xi_{j 1}^{k}=\Xi_{k 0}^{k} \frac{\psi_{j 1}^{k}}{\psi_{k 0}^{k}} .
\end{aligned}
$$

Therefore:

$$
\begin{aligned}
& \left((R P)_{k}^{j}\right)_{1}=\frac{\psi_{j 1}^{k}}{\psi_{k 0}^{k}}+\frac{A_{1}^{j} \Pi_{j 0}^{j}}{A_{0}^{k} \Pi_{k 0}^{k}}, \\
& \left((R X)_{k}^{j}\right)_{1}=\frac{\psi_{j 1}^{k}}{\psi_{k 0}^{k}}+\frac{A_{1}^{j} \Xi_{j 0}^{j}}{A_{0}^{k} \Xi_{k 0}^{k}} .
\end{aligned}
$$

The corrections to the Alfvén eigenmodes have been calculated by Andries et al. (2005b), so what remains to be computed are the corrections $A_{1}^{j}$ to the solution vectors. From the dispersion Eq. (6) in Andries et al. (2005b) expanded up to first order we get for fixed $k$ and arbitrary $j$ :

$$
\begin{aligned}
\left(\begin{array}{c}
\Pi_{j 1}^{(\mathrm{ex}, k)}-\Pi_{j 1}^{(\mathrm{in}, k)} \\
\Xi_{j 1}^{\mathrm{ex}, k)}-
\end{array}-\Xi_{j 1}^{(\mathrm{in}, k)}\right)\left(\begin{array}{c}
A_{0}^{(\mathrm{ex}, k)} \\
A_{0}^{(\mathrm{in}, k)}
\end{array}\right) \\
+\left(\begin{array}{c}
\Pi_{j 0}^{(\mathrm{ex}, j)}-\Pi_{j 0}^{(\mathrm{in}, j)} \\
\Xi_{j 0}^{(\mathrm{ex}, j)}-\Xi_{j 0}^{(\mathrm{in}, j)}
\end{array}\right)\left(\begin{array}{l}
A_{1}^{(\mathrm{ex}, j)} \\
A_{1}^{(\mathrm{in}, j)}
\end{array}\right)=0 .
\end{aligned}
$$

Thus formally:

$$
\begin{aligned}
\left(\begin{array}{c}
A_{1}^{(\mathrm{ex}, j)} \\
A_{1}^{(\mathrm{in}, j)}
\end{array}\right)= & -\left(\begin{array}{c}
\Pi_{j 0}^{(\mathrm{ex}, j)}-\Pi_{j 0}^{(\mathrm{in}, j)} \\
\Xi_{j 0}^{(\mathrm{ex}, j)}-\Xi_{j 0}^{(\mathrm{in}, j)}
\end{array}\right)^{-1} \\
& \times\left(\begin{array}{c}
\Pi_{j 1}^{(\mathrm{ex}, k)}-\Pi_{j 1}^{(\mathrm{in}, k)} \\
\Xi_{j 1}^{(\mathrm{ex}, k)}-\Xi_{j 1}^{(\mathrm{in}, k)}
\end{array}\right)\left(\begin{array}{c}
A_{0}^{(\mathrm{ex}, k)} \\
A_{0}^{(\mathrm{in}, k)}
\end{array}\right) .
\end{aligned}
$$

Using (9) and (10) we obtain:

$$
\begin{aligned}
\left(\begin{array}{l}
A_{1}^{(\mathrm{ex}, j)} \\
A_{1}^{(\mathrm{in}, j)}
\end{array}\right)=-\left(\begin{array}{l}
\Pi_{j 0}^{(\mathrm{ex}, j)}-\Pi_{j 0}^{(\mathrm{in}, j)} \\
\Xi_{j 0}^{(\mathrm{ex}, j)}-\Xi_{j 0}^{(\mathrm{in}, j)}
\end{array}\right)^{-1}\left(\begin{array}{l}
\Pi_{k 0}^{(\mathrm{ex}, k)}-\Pi_{k 0}^{(\mathrm{in}, k)} \\
\Xi_{k 0}^{(\mathrm{ex}, k)}-\Xi_{k 0}^{(\mathrm{in}, k)}
\end{array}\right) \\
\\
\times\left(\begin{array}{cc}
\psi_{j 1}^{(\mathrm{ex}, k)} / \psi_{k 0}^{(\mathrm{ex}, k)} & 0 \\
0 & \psi_{j 1}^{(\mathrm{in}, k)} / \psi_{k 0}^{(\mathrm{in}, k)}
\end{array}\right)\left(\begin{array}{l}
A_{0}^{(\mathrm{ex}, k)} \\
A_{0}^{\mathrm{in}, k)}
\end{array}\right) .
\end{aligned}
$$

We can then further rewrite the last part and apply the zeroth order dispersion relation to find:

$$
\begin{aligned}
& \left(\begin{array}{c}
A_{1}^{(\mathrm{ex}, j)} \\
A_{1}^{(\mathrm{in}, j)}
\end{array}\right)=-\left(\begin{array}{c}
\Pi_{j 0}^{(\mathrm{ex}, j)}-\Pi_{j 0}^{(\mathrm{in}, j)} \\
\Xi_{j 0}^{(\mathrm{ex}, j)}-\Xi_{j 0}^{(\mathrm{in}, j)}
\end{array}\right)^{-1}\left(\begin{array}{cc}
\Pi_{k 0}^{(\mathrm{ex}, k)}-\Pi_{k 0}^{(\mathrm{in}, k)} \\
\Xi_{k 0}^{(\mathrm{ex}, k)}-\Xi_{k 0}^{(\mathrm{in}, k)}
\end{array}\right) \\
& \times\left(\begin{array}{cc}
A_{0}^{(\mathrm{ex}, k)} & 0 \\
0 & A_{0}^{(\mathrm{in}, k)}
\end{array}\right)\left(\begin{array}{l}
\psi_{j 1}^{(\mathrm{ex}, k)} / \psi_{k 0}^{(\mathrm{ex}, k)} \\
\psi_{j 1}^{(\mathrm{in}, k)} / \psi_{k 0}^{(\mathrm{in}, k)}
\end{array}\right), \\
& =-\left(\begin{array}{ll}
\Pi_{j 0}^{(\mathrm{ex}, j)} & -\Pi_{j 0}^{(\mathrm{in}, j)} \\
\Xi_{j 0}^{\mathrm{ex}, j)} & -\Xi_{j 0}^{(\mathrm{in}, j)}
\end{array}\right)^{-1}\left(\begin{array}{c}
\Pi_{k 0}^{(\mathrm{ex}, k)}-\Pi_{k 0}^{(\mathrm{in}, k)} \\
\Xi_{k 0}^{\mathrm{ex}, k)}-\Xi_{k 0}^{(\mathrm{in}, k)}
\end{array}\right) \\
& \times\left(\begin{array}{cc}
A_{0}^{(\mathrm{ex}, k)} & -A_{0}^{(\mathrm{ex}, k)} \\
0 & 0
\end{array}\right)\left(\begin{array}{l}
\psi_{j 1}^{(\mathrm{ex}, k)} / \psi_{k 0}^{(\mathrm{ex}, k)} \\
\psi_{j 1}^{(\mathrm{in}, k)} / \psi_{k 0}^{(\mathrm{in}, k)}
\end{array}\right), \\
& =-A_{0}^{(\mathrm{ex}, k)}\left(\psi_{j 1}^{(\mathrm{ex}, k)} / \psi_{k 0}^{(\mathrm{ex}, k)}-\psi_{j 1}^{(\mathrm{in}, k)} / \psi_{k 0}^{(\mathrm{in}, k)}\right) \\
& \times\left(\begin{array}{cc}
\Pi_{j 0}^{(\mathrm{ex}, j)} & -\Pi_{j 0}^{(\mathrm{in}, j)} \\
\Xi_{j 0}^{\mathrm{ex}, j)} & -\Xi_{j 0}^{(\mathrm{in}, j)}
\end{array}\right)^{-1}\left(\begin{array}{c}
\Pi_{k 0}^{(\mathrm{ex}, k)} \\
\Xi_{k 0}^{(\mathrm{ex}, k)}
\end{array}\right) ;
\end{aligned}
$$


or equivalently:

$$
\begin{aligned}
\left(\begin{array}{l}
A_{1}^{(\mathrm{ex}, j)} \\
A_{1}^{(\mathrm{in}, j)}
\end{array}\right)= & -A_{0}^{(\mathrm{in}, k)}\left(\psi_{j 1}^{(\mathrm{ex}, k)} / \psi_{k 0}^{(\mathrm{ex}, k)}-\psi_{j 1}^{(\mathrm{in}, k)} / \psi_{k 0}^{(\mathrm{in}, k)}\right) \\
& \times\left(\begin{array}{cc}
\Pi_{j 0}^{(\mathrm{ex}, j)} & -\Pi_{j 0}^{(\mathrm{in}, j)} \\
\Xi_{j 0}^{(\mathrm{ex}, j)} & -\Xi_{j 0}^{(\mathrm{in}, j)}
\end{array}\right)^{-1}\left(\begin{array}{l}
\Pi_{k 0}^{(\mathrm{in}, k)} \\
\Xi_{k 0}^{(\mathrm{in}, k)}
\end{array}\right) .
\end{aligned}
$$

This can be solved straightforwardly by Cramer's rule:

$$
\begin{aligned}
A_{1}^{(\mathrm{ex}, j)} & =-A_{0}^{(\mathrm{ex}, k)}\left(\frac{\psi_{j 1}^{(\mathrm{ex}, k)}}{\psi_{k 0}^{(\mathrm{ex}, k)}}-\frac{\psi_{j 1}^{(\mathrm{in}, k)}}{\psi_{k 0}^{(\mathrm{in}, k)}}\right) \frac{\left|\begin{array}{l}
\Pi_{k 0}^{(\mathrm{ex}, k)}-\Pi_{j 0}^{(\mathrm{in}, j)} \\
\Xi_{k 0}^{(\mathrm{ex}, k)}-\Xi_{j 0}^{(\mathrm{in}, j)}
\end{array}\right|}{\left|\begin{array}{l}
\Pi_{j 0}^{(\mathrm{ex}, j)}-\Pi_{j 0}^{(\mathrm{in}, j)} \\
\Xi_{j 0}^{\mathrm{ex}, j)}-\Xi_{j 0}^{(\mathrm{in}, j)}
\end{array}\right|} \\
& =-\frac{A_{0}^{(\mathrm{ex}, k)} \Pi_{k 0}^{(\mathrm{ex}, k)}}{\Pi_{j 0}^{(\mathrm{ex}, j)}}\left(\frac{\psi_{j 1}^{(\mathrm{ex}, k)}}{\psi_{k 0}^{(\mathrm{ex}, k)}}-\frac{\psi_{j 1}^{(\mathrm{in}, k)}}{\psi_{k 0}^{(\mathrm{in}, k)}}\right) \frac{\left|\begin{array}{c}
1 \\
Z_{k 0}^{(\mathrm{ex}, k)}-Z_{j 0}^{(\mathrm{in}, j)}
\end{array}\right|}{\left|\begin{array}{c}
1 \\
Z_{j 0}^{(\mathrm{ex}, j)}-Z_{j 0}^{(\mathrm{in}, j)}
\end{array}\right|}, \\
& =-\frac{A_{0}^{(\mathrm{ex}, k)} \Pi_{k 0}^{(\mathrm{ex}, k)}}{\Pi_{j 0}^{(\mathrm{ex}, j)}}\left(\frac{\psi_{j 1}^{(\mathrm{ex}, k)}}{\psi_{k 0}^{(\mathrm{ex}, k)}}-\frac{\psi_{j 1}^{(\mathrm{in}, k)}}{\psi_{k 0}^{(\mathrm{in}, k)}}\right) \frac{Z_{k 0}^{\mathrm{ex}, k)}-Z_{j 0}^{(\mathrm{in}, j)}}{Z_{j 0}^{(\mathrm{ex}, j)}-Z_{j 0}^{(\mathrm{in}, j)}} .
\end{aligned}
$$

Here we have made use of the generalised impedance $Z=\Xi / \Pi$ for notational convenience. This finally enables us to write (11) and (12) as:

$$
\begin{aligned}
& \left((R P)_{k}^{\mathrm{ex} j}\right)_{1}=\frac{\psi_{j 1}^{\mathrm{ex} k}}{\psi_{k 0}^{\mathrm{ex} k}}-\left(\frac{\psi_{j 1}^{(\mathrm{ex}, k)}}{\psi_{k 0}^{(\mathrm{ex}, k)}}-\frac{\psi_{j 1}^{(\mathrm{in}, k)}}{\psi_{k 0}^{(\mathrm{in}, k)}}\right) \frac{Z_{k 0}^{(\mathrm{ex}, k)}-Z_{j 0}^{(\mathrm{in}, j)}}{Z_{j 0}^{(\mathrm{ex}, j)}-Z_{j 0}^{(\mathrm{in}, j)}} \\
& \left((R X)_{k}^{\mathrm{ex} j}\right)_{1}=\frac{\psi_{j 1}^{\mathrm{ex} k}}{\psi_{k 0}^{\mathrm{ex} k}}-\left(\frac{\psi_{j 1}^{(\mathrm{ex}, k)}}{\psi_{k 0}^{(\mathrm{ex}, k)}}-\frac{\psi_{j 1}^{(\mathrm{in}, k)}}{\psi_{k 0}^{(\mathrm{in}, k)}}\right) \frac{Z_{j 0}^{(\mathrm{ex}, j)}}{Z_{k 0}^{(\mathrm{ex}, k)}} \frac{Z_{k 0}^{(\mathrm{ex}, k)}-Z_{j 0}^{(\mathrm{in}, j)}}{Z_{j 0}^{(\mathrm{ex}, j)}-Z_{j 0}^{(\mathrm{in}, j)}}
\end{aligned}
$$

The impedances can be entirely expressed in terms of the Alfvén eigenvalues $\lambda_{j 0}^{\mathrm{i}, \mathrm{e}}$ and two parameters: the radius $R$ and the azimuthal wave number $m$. The expressions involve Bessel functions for which the small argument expansions can be used in the limit of a "thin tube" which reduces them to the simple expression:

$Z_{n 0}^{n}=\frac{\Xi_{n 0}^{n}}{\Pi_{n 0}^{n}} \approx \pm \frac{m}{R} \lambda_{n 0}^{-1}$.

Here and in what follows the upper sign applies for the internal expressions (with superscript in) and the lower sign for the external expressions (with superscript ex). The corrections to the Alfvén eigenfunctions can also be expressed in terms of the Alfvén eigenvalues $\lambda_{j 0}^{\mathrm{i}, \mathrm{e}}$ as was obtained by Andries et al. (2005b):

$$
\frac{\psi_{j 1}^{\mathrm{in}, \mathrm{ex} k}}{\psi_{k 0}^{\mathrm{in}, \mathrm{ex} k}}=-\frac{\rho_{0} \omega^{2}}{\lambda_{j 0}^{\mathrm{in}, \mathrm{ex}}-\lambda_{k 0}^{\mathrm{in}, \mathrm{ex}}} \alpha S_{k j} .
$$

Thus the expressions for the ratios (23) and (24) can be expressed solely in terms of the Alfvén eigenvalues $\lambda_{j 0}$. Therefore let us write out $\lambda_{j 0}^{\text {in,ex }}$ explicitly. Care has to be taken here as the frequency in the expressions for $\lambda_{j 0}$ is nevertheless that of the $k$ th mode. Hence, using the expression for the kink frequency:

$$
\begin{aligned}
\lambda_{j 0}^{\mathrm{in}, \mathrm{ex}} & =\rho_{\mathrm{in}, \mathrm{ex}}\left(\omega_{\mathrm{k}}^{2}+v_{\mathrm{A}}^{2}\left(\frac{j \pi}{L}\right)^{2}\right)=\left(\left(\rho_{\mathrm{in}, \mathrm{ex}} \frac{2 k^{2}}{\rho_{\mathrm{i}}+\rho_{\mathrm{e}}}\right)-j^{2}\right)\left(\frac{B \pi}{L}\right)^{2} \\
& =\left((1 \pm r) k^{2}-j^{2}\right)\left(\frac{B \pi}{L}\right)^{2}=\left(k^{2}-j^{2} \pm r k^{2}\right)\left(\frac{B \pi}{L}\right)^{2} .
\end{aligned}
$$

Here $r$ is a parameter related to the density contrast:

$r=\frac{\rho_{\mathrm{i}}-\rho_{\mathrm{e}}}{\rho_{\mathrm{i}}+\rho_{\mathrm{e}}}$.

A fortiori we have:

$\lambda_{k 0}^{\text {in,ex }}= \pm r k^{2}\left(\frac{B \pi}{L}\right)^{2}$,

and thus for the corrections to the eigenfunctions:

$\frac{\psi_{j 1}^{\mathrm{in}, \mathrm{ex} k}}{\psi_{k 0}^{\mathrm{in}, \mathrm{ex} k}}=\frac{\left(\rho_{\mathrm{i}, \mathrm{e}} \frac{2 k^{2}}{\rho_{\mathrm{i}}+\rho_{\mathrm{e}}}\right)\left(\frac{B \pi}{L}\right)^{2}}{\lambda_{k 0}^{\mathrm{i}, \mathrm{e}}-\lambda_{j 0}^{\mathrm{i}, \mathrm{e}}} \alpha S_{k j}=(1 \pm r) \frac{k^{2}}{j^{2}-k^{2}} \alpha S_{k j}$

By use of the approximations for the impedances (25), the ratios (23) and (24) therefore become:

$$
\begin{aligned}
\left((R P)_{k}^{\mathrm{ex} j}\right)_{1} & =\left\{(1-r) \frac{k^{2}}{j^{2}-k^{2}}-\frac{-2 r k^{2}}{j^{2}-k^{2}} \frac{k^{2}-j^{2}-r k^{2}}{-r k^{2}} \frac{1}{2}\right\} \alpha S_{k j} \\
& =\frac{j^{2}}{j^{2}-k^{2}} \alpha S_{k j} \\
\left((R X)_{k}^{\mathrm{ex} j}\right)_{1} & =\left\{(1-r) \frac{k^{2}}{j^{2}-k^{2}}-\frac{-2 r k^{2}}{j^{2}-k^{2}} \frac{1}{2}\right\} \alpha S_{k j} \\
& =\frac{k^{2}}{j^{2}-k^{2}} \alpha S_{k j} .
\end{aligned}
$$

It is straightforward to check that Eq. (32) is equivalent to that obtained by Safari et al. (2007).

\section{Discussion of the analytical results}

The expressions for the amplitude ratios (31-32) nicely summarise the coupling of the different axial Fourier components in the kink-eigenfunctions in (weakly) stratified loops. In general $\alpha S_{k j}$ is actually a shorthand notation for $\sum_{n} \alpha_{n} S_{n k j}$. When a specific stratification is chosen which depends on a single stratification parameter, then in a linear expansion the alphas are simply proportional to the stratification parameter. If the stratification is sufficiently smooth, $\alpha_{n}$ quickly becomes smaller with $n$ and retaining only the $n=1$ contribution is a fairly good approximation for many profiles. In fact for the specific profile of an exponential atmosphere with scale height $H$ projected on a semicircular loop (as used by Andries et al. 2005a; Verth et al. 2007), all alphas except $\alpha_{1}$ are second order and $\alpha_{1}=-L / \pi H$.

The values of the first 6 coefficients of the sine expansions (linear in $\alpha$ ) of the first 5 modes are summarised in Table 1. The diagonal entries are set to 1 and in absolute value at least a factor of 3 larger than the off-diagonal entries. Several interesting conclusions may be drawn, both from the table and from the expressions. Firstly, the contributions of the off-diagonal terms become stronger as $k$ increases, both in displacement and compression. Hence, it seems that if higher order axial overtones could be detected, their potential for spatial seismology will be higher. Secondly, for the $k$ th mode the largest coupling in the compression is always with the $(k+2)$ th sine, while the $(k-2)$ th contribution also becomes significant for higher $k$ (and eventually of the same order). In the displacement it is the $(k-2)$ th term that dominates with the $(k+2)$ th sine almost of the same order (and dominating for $k<3$ as the $(k-2)$ th term is absent there). Thus for the two modes observed so far $(k=1,2)$ the behaviour can almost completely be characterised by the coupling with the third and fourth sine contribution respectively. Furthermore, the relative effect of longitudinal density stratification is always stronger 
Table 1. Sine expansions of the first 5 eigenmodes $(k)$ when expanded linearly in the stratification parameter.

\begin{tabular}{c|cccccc}
\hline \hline \multicolumn{7}{c}{ Displacement: } \\
\hline$k j$ & 1 & 2 & 3 & 4 & 5 & 6 \\
\hline 1 & 1 & 0 & -.021221 & 0 & -.001011 & 0 \\
2 & 0 & 1 & 0 & -.064672 & 0 & -.004042 \\
3 & 0.190986 & 0 & 1 & 0 & -.113682 & 0 \\
4 & 0 & 0.258690 & 0 & 1 & 0 & -.164621 \\
5 & 0.025263 & 0 & 0.315784 & 0 & 1 & 0 \\
\hline \hline \multicolumn{7}{c}{ Compression: } \\
\hline$k j$ & 1 & 2 & 3 & 4 & 5 & 6 \\
\hline 1 & 1 & 0 & -.190986 & 0 & -.025263 & 0 \\
2 & 0 & 1 & 0 & -.258690 & 0 & -.036378 \\
3 & 0.021221 & 0 & 1 & 0 & -.315784 & 0 \\
4 & 0 & 0.064672 & 0 & 1 & 0 & -.370397 \\
5 & 0.001011 & 0 & 0.113682 & 0 & 1 & 0 \\
\hline
\end{tabular}

The off-diagonal numbers (i.e. $\left((R X)_{k}^{\mathrm{ex} j}\right)_{1}$ and $\left.\left((R P)_{k}^{\mathrm{ex} j}\right)_{1}\right)$ are the coefficients of $\alpha_{1}$ in the linear expansion.

Table 2. Shift of the antinodes $(i)$ of the first 5 eigenmodes $(k)$ when expanded linearly in the stratification parameter.

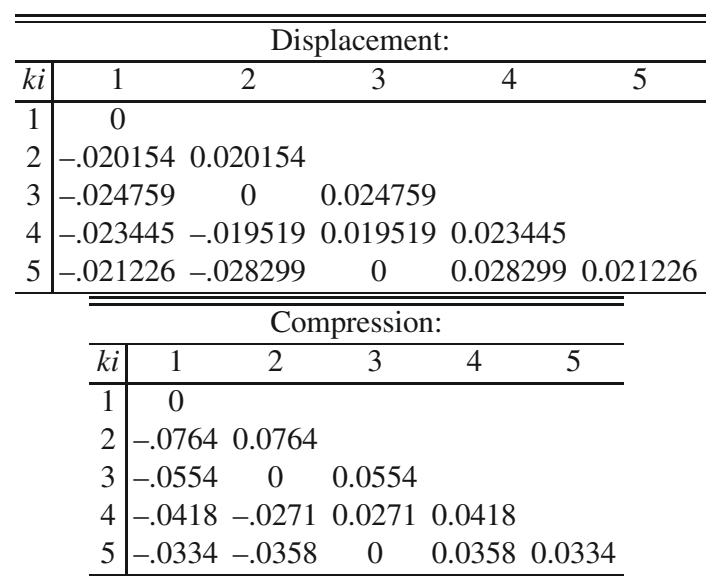

The numbers are the coefficients of $\alpha_{1}$ in the expansion. Note the number of digits in the table for the compression. The terms in summation (33) decay rather slowly with $j$ and although 200 terms were used to compute the above values, they are only valid up to the third significant digit. For the displacement the contributions decay much faster with $j$ and are almost entirely determined by the $(k+2)$ th contribution.

in the compression than in the lateral displacement by a factor $(k+2)^{2} / k^{2}$. Hence the presence of the $j=3$ Fourier contribution in the fundamental oscillation is 9 times more pronounced in the total pressure perturbation than in the displacement, and the modifications to the first overtone are expected to be 4 times larger in the compression than in the displacement.

These results in terms of additional sine contributions in the eigenmodes can straightforwardly be related to the spatial shifts of the antinodes as investigated by Verth et al. (2007). From a linear expansion we find that the shift of the $i$ th antinode can be approximated as:

$\frac{\Delta z_{i}}{L}=(-1)^{i} \sum_{j}\left((R X)_{k}^{\mathrm{ex} j}\right)_{1} \frac{j}{k^{2} \pi} \cos \left(\frac{j \pi}{k}\left(i-\frac{1}{2}\right)\right)$.

The results for all the antinodes of the first 5 modes are given in Table 2. Although for higher harmonics the contributions of the other Fourier modes increase, the shift of the antinodes remains roughly at the same order independent of the harmonic. It thus seems that the shifts of the antinodes are not the best signatures of the eigenmode modifications. Note that this is not due to the fact that the $(k+2)$ th sine and the $(k-2)$ th sine have opposite sign, as this is corrected for by the cosine factor in the expression for the shift (33). It can be argued that the shift should be normalised to the wavelength (varying as $1 / k$ ) and not to the length of the loop, in which case the conclusion would be that the shift increases linearly with $k$. From an observational point however it would be appropriate to normalise with respect to the resolution, which evidently does not depend on $k$. It can also be seen that the shift of the antinodes for the first overtone is as expected roughly about 4 times stronger in the compression than in the displacement. For higher harmonics the difference in the effect on displacement and compression also becomes smaller, as expected. However this does not follow the simple dependence $(k+2)^{2} / k^{2}$ derived above as the contributions from $j \neq k+2$ in the sum (33) are not negligible compared to the contribution of $j=k+2$. This particularly affects the shift of the antinode in the compression, where contributions are significant over a large range of $j$.

The predicted shift in the antinode of the first overtone can be compared with the results by Verth et al. (2007). They normalise to half of the loop length and use $L / H$ (thus actually $L / 2 H$ ) rather than $L / \pi H$ as a stratification parameter, hence for comparison their proportionality constants should be multiplied by $\pi / 4$. Their estimation based on the analytical model, which as in our treatment involves a linearisation in the stratification parameter, matches exactly with our results, and their numerical results deviated no more than $11 \%$ from that value.

\section{Comparison with direct numerical computations}

The correspondence with the results by Verth et al. (2007) is reassuring, but to gain even more confidence in the acquired results we have compared them with direct numerical eigenmode computations (i.e. non-linear in the stratification parameter) by means of the PDE2D code (Sewell 2005), a general-purpose partial differential equation solver. Figure 1 shows the shifts of the antinodes as a function of the stratification parameter $\alpha$ in a model with a single fundamental Fourier contribution to the longitudinal density variation. The panels are for the first and second overtone and for both displacement and compression. It can be seen that the analytical prediction is indeed a valid first order approximation of the obtained results. However, for 

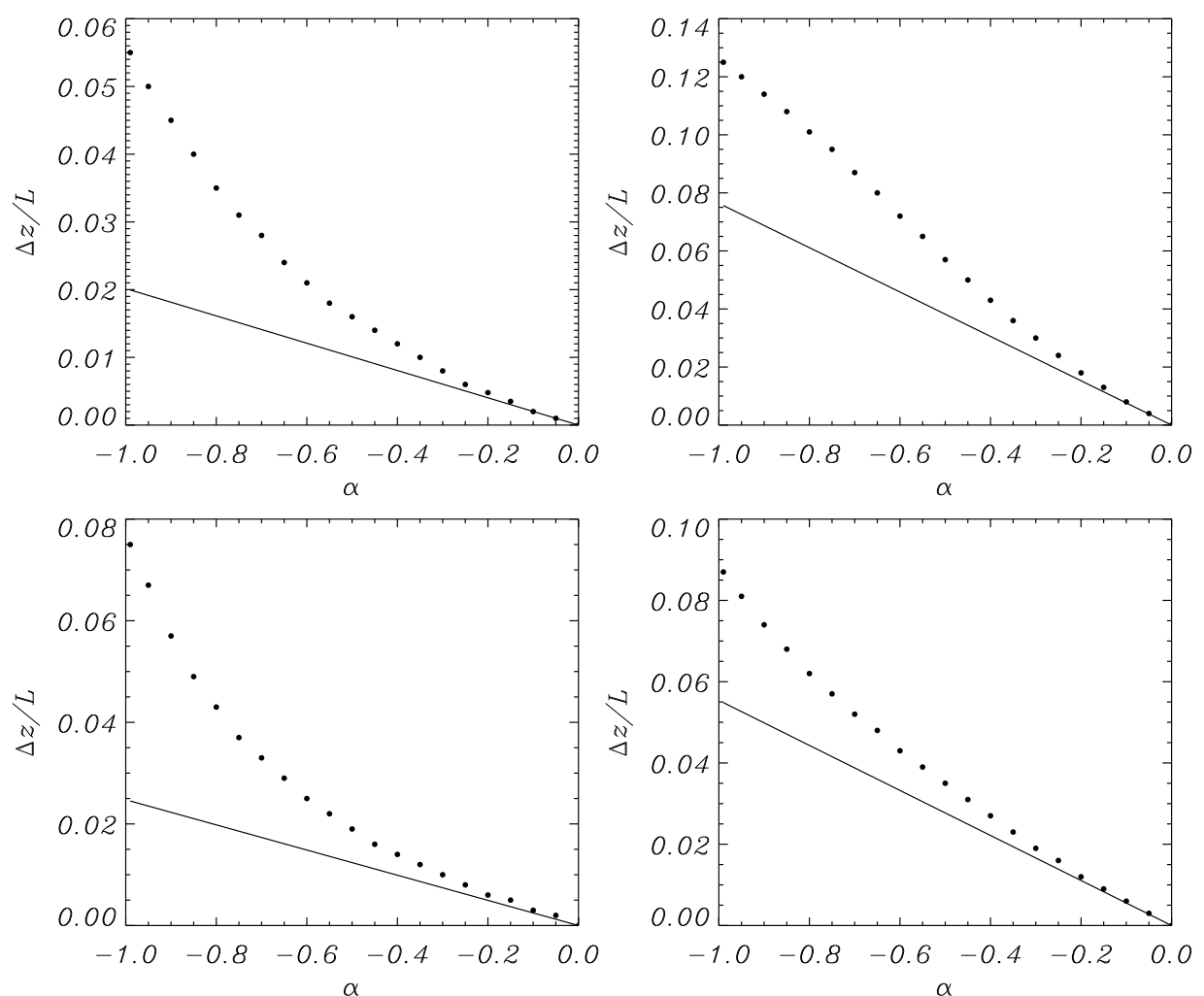

Fig. 1. Shift of the antinodes as a function of the stratification parameter $\alpha$ in a model using the density stratification function $\rho(r, z)=$ $\rho_{0}(r)[1+\alpha \sin (\pi z / L)]$. Dots are results of direct numerical calculation while full lines correspond to the analytical approximations for small stratification. Upper and lower panels are for the first and second overtone respectively. In the left panels the eigenfunction of the displacement is considered while the compression is considered in the right panels.
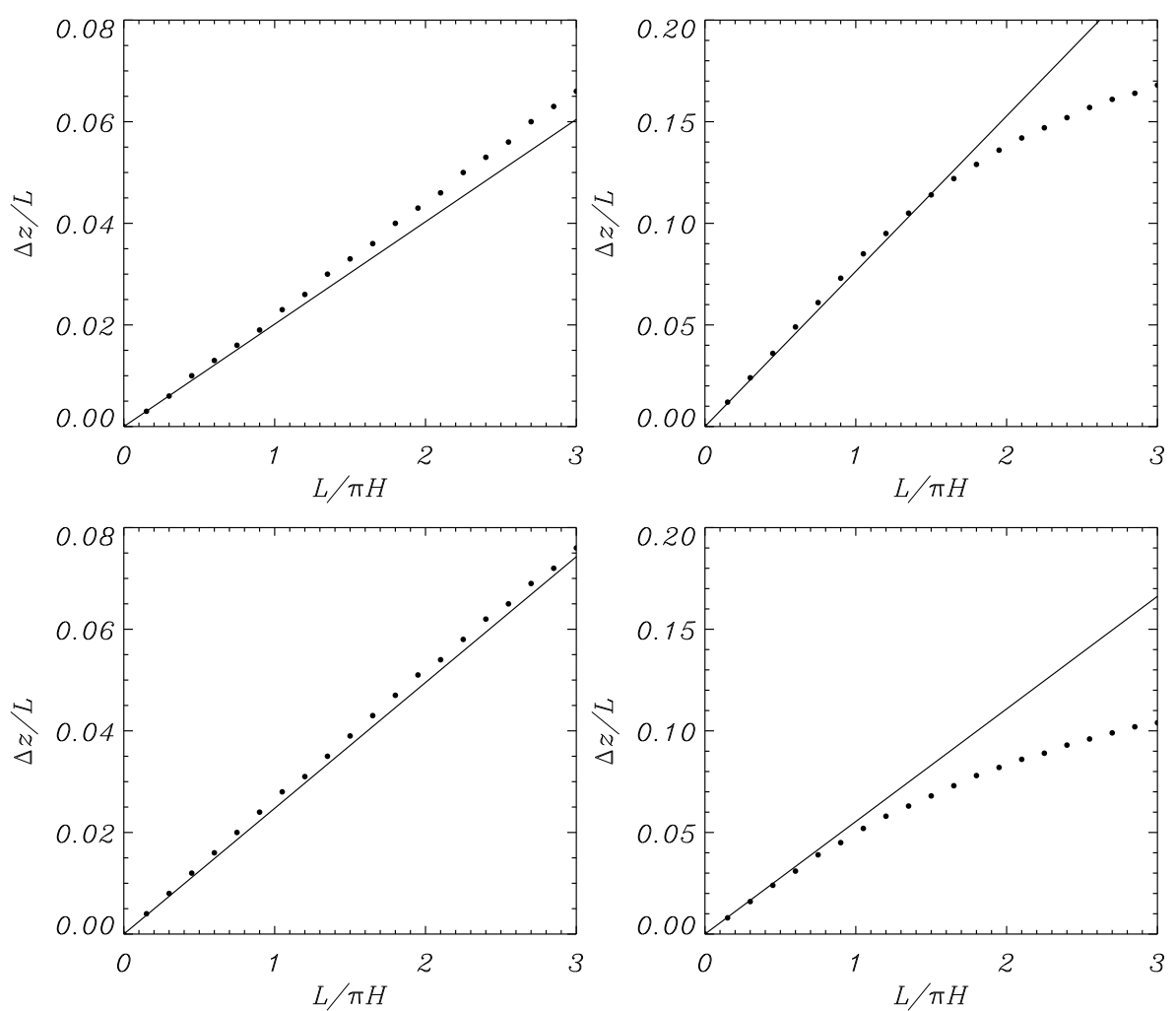

Fig. 2. Shift of the antinodes as a function of the stratification parameter $L / \pi H$ in a model using the density stratification function $\rho(r, z)=$ $\rho_{0}(r) \exp [-L / \pi H \sin (\pi z / L)]$. Dots are results of direct numerical calculation while full lines correspond to the analytical approximations for small stratification. Upper and lower panels are for the first and second overtone respectively. In the left panels the eigenfunction of the displacement is considered while the compression is considered in the right panels.

non-linear values of $\alpha$, say above 0.4 , the results already differ from the linear predictions by more than $20 \%$. This is surprising, as Verth et al. (2007) had obtained a fairly linear behaviour throughout. In Fig. 2, the model density variation was taken as by Andries et al. (2005a); Verth et al. (2007). i.e. an exponential atmosphere with scale height $H$ projected on a semicircular loop: $\rho(r, z)=\rho_{0}(r) \exp [-L / \pi H \sin (\pi z / L)]$. In a linear approximation both models are equivalent and $\alpha=-L / \pi H$ as mentioned earlier. Indeed, also here the linear prediction can be seen to be valid. However, for larger values the two models do differ significantly. For the shift in the antinodes of the displacement again a fairly linear behaviour is found throughout the parameter domain although with a slightly increased slope (as also obtained by Verth et al. 2007). This is less so for the antinodes in the compression. It is striking that the model with the exponential profile behaves much more linearly than the model 

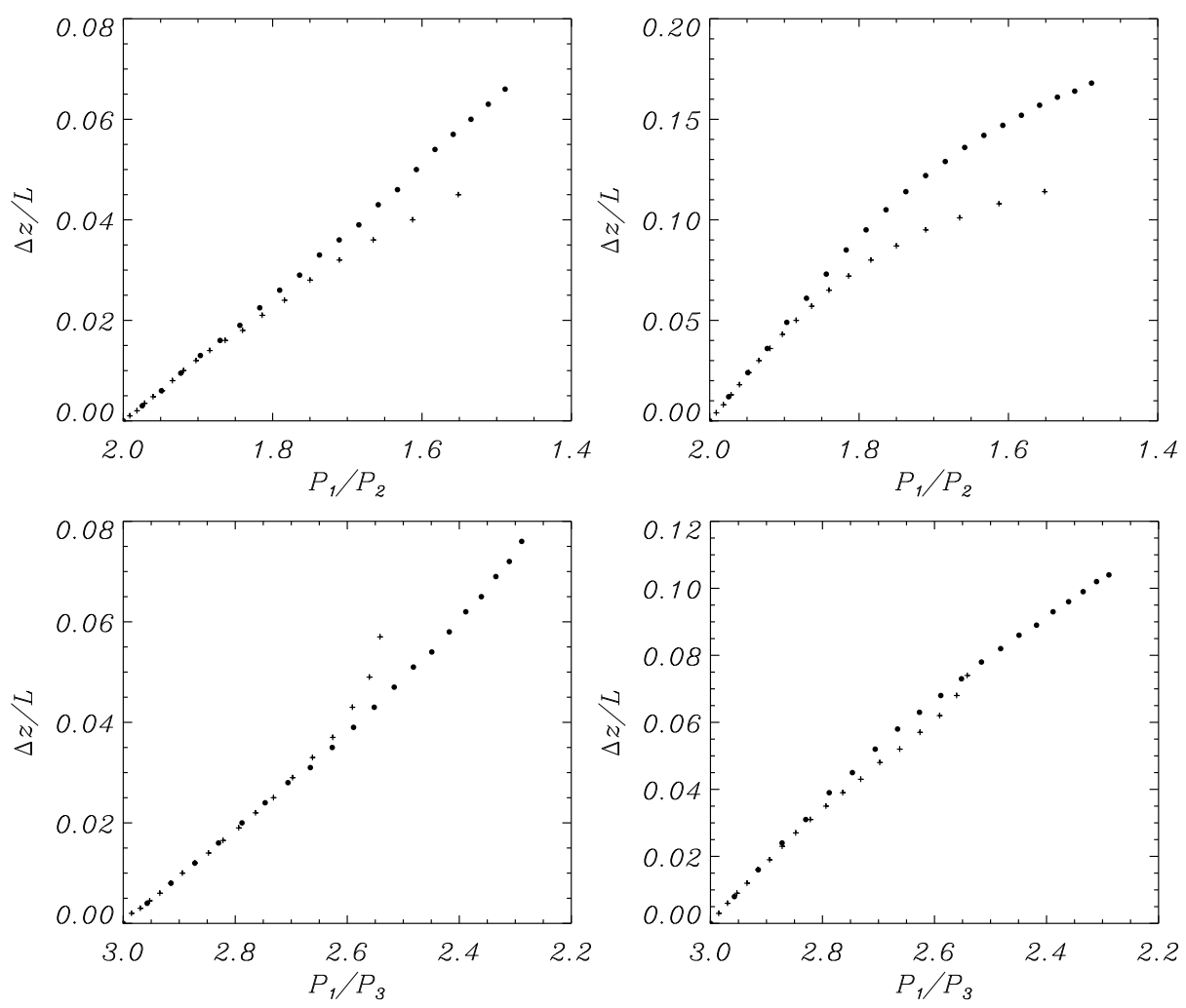

Fig. 3. Shift of the antinodes as a function of the period ratio $P_{1} / P_{2}$ and $P_{1} / P_{3}$ respectively. Dots correspond to the model with $\rho(r, z)=\rho_{0}(r) \exp [-L / \pi H \sin (\pi z / L)]$ while crosses are for the model $\rho(r, z)=\rho_{0}(r)[1+\alpha \sin (\pi z / L)]$. Upper and lower panels are for the first and second overtone respectively. In the left panels the eigenfunction of the displacement is considered while the compression is considered in the right panels.

with the sinusoidal stratification, on which the linear approximation is actually based.

Also note that the figures indicate that the difference between the results of the two models is not only due to the nonlinear relation between $\alpha_{1}$ and $L / \pi H$ but also because of the nonvanishing values of $\alpha_{3}, \alpha_{5}, \ldots$ This can be clearly seen by looking up, for a certain value of $\alpha$, the shifts for both displacement and compression (in Fig. 1), and then finding the corresponding values of $L / \pi H$ in the second model (through Fig. 2). In this way the values for $L / \pi H$ are found to be different when considering displacement or compression. Hence, the results in the two models do not correspond through a simple mapping between the parameters $\alpha$ and $L / \pi H$.

With regard to the potential of using the observed eigenfunctions (or antinode shifts) the following question is pertinent. If the period ratio $P_{2} / P_{1}$ (or $P_{3} / P_{1}$ ) is observed accurately, does observation of the antinode shift allow us to discriminate between the two models? Hence Figs. 1 and 2 are repeated in Fig. 3 but as a function of the corresponding period ratio, which allows us to make a clear comparison between the different models, based on two observational parameters. As can be seen, a highly deviating period ratio is required before the antinode shifts between the models differ substantially. This is to be expected for the two models used here as they only differ in second order. Similarly in Fig. 4 the eigenfunctions are plotted. One curve represents the solution in the unstratified case, while the other two curves are the solutions in the two different models, where the stratification parameters are such that the resulting period ratios are equal in both models. Again it can be seen that the modifications are not significantly different between the two models considered. Much more significant differences between the models can only be expected if there is an important contribution of higher order sines in the stratification profile, hence if there are more sudden longitudinal changes in the density, rather than the smooth profiles that are considered here.

\section{Conclusions}

This paper is aimed at assessing the possibility that higher overtones of coronal loop kink modes might be more usefull for spatial seismology. By means of a linear expansion in the longitudinal stratification along with the "thin tube" approximation, the modifications to the eigenfunctions are calculated analytically. Simple and concise formulae are obtained, representing the $j$ th Fourier contribution in the $k$ th eigenmode. The results also provide a linear estimate of the shift of the antinodes. It is found that the higher overtones are indeed influenced even more than the first overtone, and that for the first overtones the influence is much stronger in the compression than in the transverse displacement. Also, the shift of the anti-nodes does not seem to be the best signature of the eigenmode modifications.

The results are finally compared with direct numerical eigenmode computations. Although, the linear results give a good indication of the order of magnitude of the modifications, the deviations from the analytical predictions can certainly not be neglected under the expected coronal conditions. Although the results differ between models, it is clarified that if models are considered with smooth density profiles which hence have a dominant contribution from the fundamental sine in the stratification function, it is unlikely that the differences can be probed by the observational determination of the eigenfunctions. The numerical results indicate that the use of the eigenmode modifications as a tool to discriminate between two different but smooth density models requires both a very stratified equilibrium and a highly accurate determination of the anti-node shifts.

Acknowledgements. J.A. was supported by an International Outgoing Marie Curie Fellowship within the 7th European Community Framework Programme. 

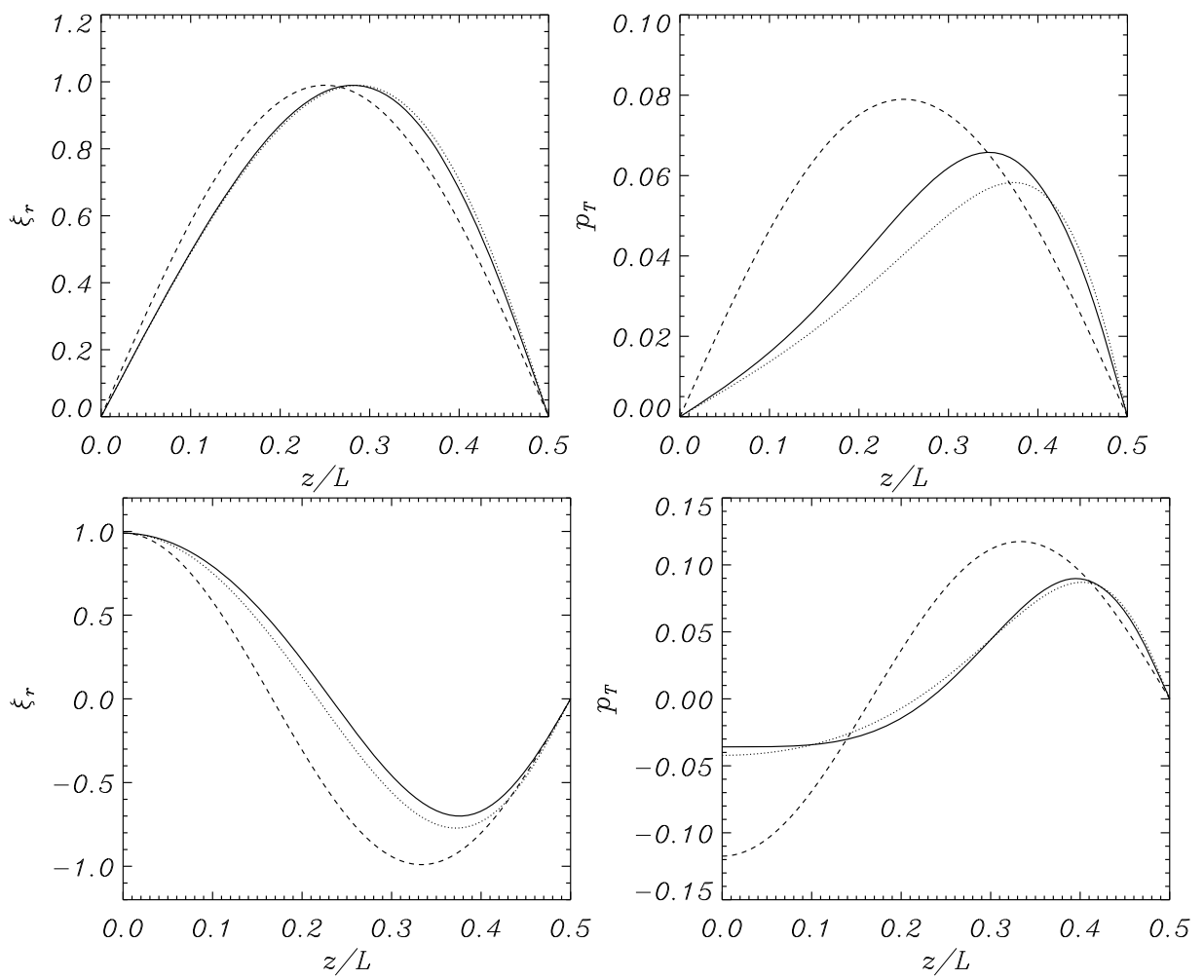

Fig. 4. Eigenfunctions of the first (upper panels) and second (lower panels) overtones. Left is the displacement, right is the compression. Full lines correspond to the eigenfunctions obtained for the model $\rho(r, z)=\rho_{0}(r)[1+\alpha \sin (\pi z / L)]$. The dotted curves are for the model $\rho(r, z)=\rho_{0}(r) \exp [-L / \pi H \sin (\pi z / L)]$. The parameters in both models are chosen such that they lead both to a period ratio of 1.71 and 2.59 respectively. Dashed lines are the reference functions in a model without longitudinal density variation.

I.A. acknowledges the support from the grant AYA2006-07637. M.G. acknowledges support from the grant GOA/2009-009.

\section{References}

Andries, J., Arregui, I., \& Goossens, M. 2005a, ApJ, 624, L57

Andries, J., Goossens, M., Hollweg, J., Arregui, I., \& Van Doorsselaere, T. 2005b, A\&A, 430, 1109

Arregui, I., Van Doorsselaere, T., Andries, J., \& Goossens, M. 2007, A\&A, 463, 333

De Moortel, I., \& Brady, C. 2007, ApJ, 664, 1210

Díaz, A. J., Oliver, R., \& Ballester, J. L. 2002, ApJ, 580, 550

Díaz, A. J., Oliver, R., Ballester, J. L., \& Roberts, B. 2004, A\&A, 424, 1055

Díaz, A. J., Oliver, R., \& Ballester, J. 2006, ApJ, 645, 766

Díaz, A. J., Donnelly, G. R., \& Roberts, B. 2007, A\&A, 476, 359

Donnelly, G. R., Díaz, A. J., \& Roberts, B. 2006, A\&A, 457, 707

Dymova, M., \& Ruderman, M. 2005, Sol. Phys., 229, 79

Dymova, M., \& Ruderman, M. 2006a, A\&A, 459, 241

Dymova, M., \& Ruderman, M. 2006b, A\&A, 457, 1059

Edwin, P. M., \& Roberts, B. 1983, Sol. Phys., 88, 179

Erdélyi, R., \& Verth, G. 2007, A\&A, 462, 743
Goossens, M., Andries, J., \& Aschwanden, M. 2002, A\&A, 394, L39 Goossens, M., Arregui, I., Ballester, J., \& Wang, T. 2008, A\&A, 484, 851 Jess, D., Mathioudakis, M., Erdélyi, R., et al. 2008, ApJ, 680, 1523

McEwan, M., Donnelly, G., Díaz, A., \& Roberts, B. 2006, A\&A, 460, 893 McEwan, M., Díaz, A., \& Roberts, B. 2008, A\&A, 481, 819

Nakariakov, V. M. 2000, in AIP Conf. Proc., 537, 264

Nakariakov, V. M., \& Ofman, L. 2001, A\&A, 372, L53

Nakariakov, V. M., \& Verwichte, E. 2005, Living Rev. Sol. Phys., 2, 3

Roberts, B. 2008, in IAU Symp., 247, 3

Ruderman, M. S. 1992, J. Plas. Phys., 47, 175

Ruderman, M. S., \& Roberts, B. 2002, ApJ, 577, 475

Ruderman, M. S., Verth, G., \& Erdélyi, R. 2008, ApJ, 686, 694

Safari, H., Nasiri, S., \& Sobouti, Y. 2007, A\&A, 470, 1111

Sewell, G. 2005, The Numerical Solution of Ordinary and Partial Differential Equations (Wiley-Interscience)

Van Doorsselaere, T., Nakariakov, V., \& Verwichte, E. 2007, A\&A, 473, 959

Verth, G., \& Erdélyi, R. 2008, A\&A, 486, 1015

Verth, G., Van Doorsselaere, T., Erdélyi, R., \& Goossens, M. 2007, A\&A, 475, 341

Verth, G., Erdélyi, R., \& Jess, D. B. 2008, ApJ, 687, L41

Verwichte, E., Nakariakov, V., Ofman, L., \& Deluca, E. 2004, Sol. Phys., 223, 77 